\title{
Prevalência e fatores de risco da doença arterial periférica sintomática e assintomática em hospital terciário, Rio de Janeiro, Brasil
}

\author{
Prevalence and risk factors of symptomatic and asymptomatic \\ peripheral arterial disease in a tertiary care hospital, \\ Rio de Janeiro, Brazil
Marilia Duarte Brandão Panico ${ }^{1}$, Ethel Stambovsky Spichler ${ }^{2}$, Mario Fritsch Neves ${ }^{3}$, Liana Wernersbach Pinto ${ }^{4}$, David Spichler ${ }^{5}$

\section{Resumo}

Contexto: Doença arterial periférica é caracterizada pelo índice tornozelo-braquial (ITB) $<0,90$, em indivíduos $\geq 40$ anos, aumentando a prevalência com a idade.

Objetivo: Detectar a prevalência de doença arterial periférica assintomática e sintomática, com introdução do ITB, associada a fatores de risco demarcados.

Métodos: Coorte descritiva identificada em unidade hospitalar terciária de angiologia, de dezembro de 2006 a dezembro de 2007, com idade $\geq 30$ anos. Doenças pregressas e fatores de risco foram analisados associados à prevalência. ITB $<0,90$ e questionário padronizado definiram doença arterial periférica sintomática com claudicação e assintomática com ausência de claudicação, ambas comparadas aos sem doença arterial periférica (ITB 0,90-1,30). A análise estatística utilizou programa SPSS, com significância de $\mathrm{p}<0,05$.

Resultados: Dos 407 pacientes, 248 apresentaram doença arterial periférica, sendo $54 \%$ do sexo feminino, com média de idade de 70,1 $\pm 10,2$ anos $(\mathrm{p}<0,005)$. A prevalência de 60,9\% (IC95\% 56-66) foi subdividida em: assintomática, 10,1\% (IC95\% 6,3-13,8); e sintomática, 89,9\% (IC95\% 86,2-93,7). Destes, 32,2\% (IC95\% 26,4-38,1) apresentaram isquemia crítica. Ajustada por sexo e idade, a prevalência aumenta significativamente entre 55-74 anos, com predomínio do feminino (1,35:1) nos indivíduos acima de 74 anos. A prevalência dos assintomáticos e sintomáticos foi influenciada por tabagismo, hipertensão, diabéticos autorreferidos e confirmados, sobrepeso, infarto agudo do miocárdio e acidente vascular encefálico $(\mathrm{p}<0,005)$. A média do ITB foi mais baixa nos sintomáticos $(0,57 \pm 0,17)(\mathrm{p}<0,005)$.

Conclusão: O ITB detectou doença arterial periférica com graus variáveis de gravidade associada a fatores de risco, identificando os assintomáticos não-claudicantes e os sintomáticos em unidade terciária.

Palavras-chave: Doença arterial periférica sintomática e assintomática, diagnóstico, índice tornozelo-braquial, prevalência, fatores de risco.

\begin{abstract}
Background: Peripheral arterial disease (PAD) is defined as an anklebrachial index $(\mathrm{ABI})<0.90$ in individuals aged 40 years or over. Prevalence is known to increase with age.

Objective: To detect the prevalence of asymptomatic and symptomatic $\mathrm{PAD}$, using $\mathrm{ABI}$, in association with risk factors.

Methods: A descriptive cohort of patients $\geq 30$ years old was identified at the outpatient angiology clinic of a tertiary referral center, from December 2006 to December 2007. Previous pathologies and risk factors were analyzed in relation to PAD prevalence. ABI $<0.90$ and a specific questionnaire defined symptomatic PAD with claudication, and asymptomatic PAD without claudication, both in comparison with patients without PAD (ABI 0.90-1.30). Statistical analyses were performed with SPSS, considering $\mathrm{p}<0.05$.

Results: Of the 407 patients, 248 had PAD; $54 \%$ were females, with a mean age of $70.1 \pm 10.2$ years $(\mathrm{p}<0.005)$. PAD prevalence was $60.9 \%(95 \%$ CI $56-66)$, divided as follows: asymptomatic, $10.1 \%$ (95\% CI 6.3-13.8); and symptomatic, $89.9 \%$ (95\%CI 86.2-93.7). Among the symptomatic patients, 32.2\% (95\%CI 26.4-38.1) presented critical ischemia. Age- and sex-adjusted analyses revealed a dramatically increased prevalence in patients aged 55-74 years, with a predominance of female patients aged $>74$ years $(1.35: 1)$. The prevalence of asymptomatic and symptomatic PAD was affected by smoking, hypertension, diabetes (both self-reported and confirmed), obesity, acute coronary heart disease and stroke $(\mathrm{p}<0.005)$. Mean ABI was lower in symptomatic PAD $(0.57 \pm 0.17)(\mathrm{p}<0.005)$.
\end{abstract}

Conclusion: $\mathrm{ABI}$ was able to detect PAD with variable degrees of severity, associated with risk factors, by identifying symptomatic and asymptomatic PAD patients at a tertiary center.

Keywords: Peripheral arterial disease, symptomatic, asymptomatic, diagnostic, ankle-brachial index, prevalence, risk factors.

1. Chefe, Disciplina de Angiologia, Faculdade de Ciências Médicas, Hospital Universitário Pedro Ernesto (HUPE), Universidade do Estado do Rio de Janeiro (UERJ), Rio de Janeiro, RJ.

2. Professora titular visitante, Faculdade de Ciências Médicas, HUPE, UERJ, Rio de Janeiro, RJ.

3. Doutor. Professor e chefe, Departamento de Clínica Médica, Faculdade de Ciências Médicas, HUPE, UERJ, Rio de Janeiro, RJ.

4. Pesquisadora, Saúde Pública, Centro Latino-Americano de Estudos de Violência e Saúde Jorge Careli (CLAVES), Escola Nacional de Saúde Pública (ENSP), Fundação Oswaldo Cruz (FIOCRUZ), Rio de Janeiro, RJ.

5. Livre-docente, Cirurgia Vascular, Universidade Federal do Estado do Rio de Janeiro (UNIRIO), Rio de Janeiro, RJ.

Trabalho realizado na Disciplina de Angiologia, Faculdade de Ciências Médicas, Hospital Universitário Pedro Ernesto (HUPE), Universidade Estadual do Rio de Janeiro (UERJ), Rio de Janeiro, RJ.

Não foram declarados conflitos de interesse associados à publicação deste artigo.

Artigo submetido em 01.09.08, aceito em 15.01.09.

J Vasc Bras. 2009;8(2):125-132.

Copyright (C 2009 by Sociedade Brasileira de Angiologia e de Cirurgia Vascular 


\section{Introdução}

A doença arterial periférica (DAP) atinge 8 milhões de americanos, com claudicação intermitente. Para o seu diagnóstico, utiliza-se o índice tornozelo-braquial (ITB) $<0,90$, método eficaz também na sua progressão $0^{1-3}$. No entanto, 20 a $50 \%$ são detectados como assintomáti$\cos ^{4}$.

Tal panorama, descrito por Weitz et al. ${ }^{5}$, foi endossado por diversos estudos ${ }^{4,6-9}$. Esses autores delinearam a progressão da DAP expressa abaixo na Figura 1, atualizada por Hirsch et al. ${ }^{4}$.

A incidência de amputações no Rio de Janeiro, como desfecho desfavorável, apresentou taxas de 31/100.000 habitantes, 209/100.000 diabéticos e 359/100.000 com DAP, sendo esta a principal etiologia, com $56,4 \%$ entre indivíduos de 65 anos $^{10,11}$. A acurácia diagnóstica comparou o ITB com o padrão-ouro, a angiografia, concluindo pela superioridade do primeiro na informação hemodinâmica versus morfológica ${ }^{12}$.

\section{Objetivo}

Detectar a prevalência e fatores de risco da DAP sintomática e assintomática, com introdução do ITB, associada a fatores de risco demarcados.

\section{Métodos}

A população do estudo, uma coorte descritiva, foi identificada na Unidade de Angiologia do Hospital Universitário Pedro Ernesto (HUPE), da Universidade do Estado do Rio de Janeiro (UERJ), no período de dezembro de 2006 a dezembro de 2007. O projeto foi submetido e aprovado pelo Comitê de Ética em Pesquisa do HUPE, com esclarecimentos e termo de consentimento informado.

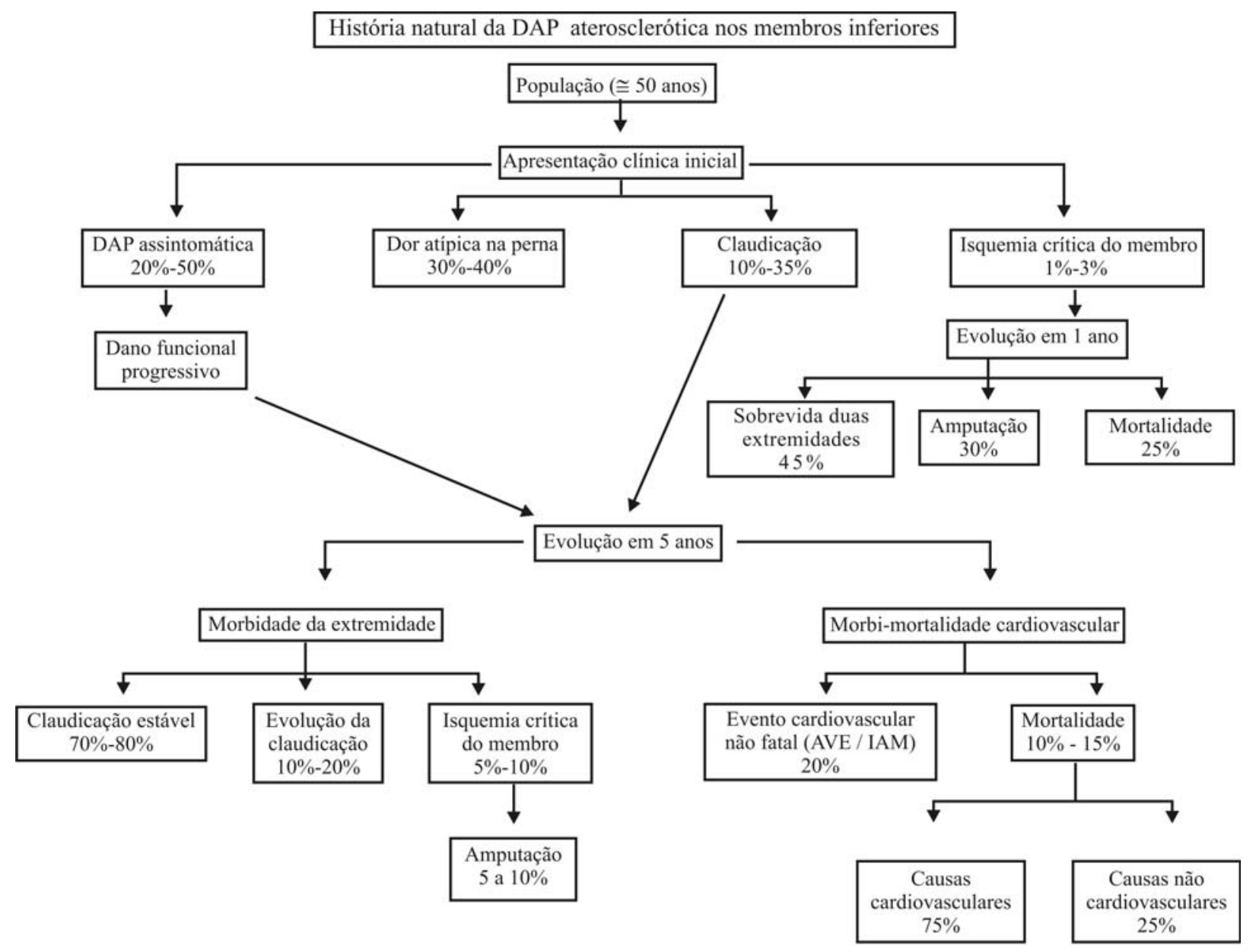

$\mathrm{AVE}=$ acidente vascular encefálico DAP $=$ doença arterial periférica; $\mathrm{IAM}=$ infarto agudo do miocárdio.

Figura 1 - História natural da DAP aterosclerótica nos membros inferiores 
O critério de inclusão predefiniu idade $\geq 30$ anos e o perfil de fatores de risco ${ }^{13}$, excluídas as pseudoclaudicações $^{14}$. Idade (estratificada, 30-54, 55-74 e > 74), sexo, cor da pele (branca, não-branca), tabagismo (fumantes, ex-fumantes e não-fumantes), hipertensão e diabetes foram autorreferidos. Doenças pregressas cardiovasculares, como infarto agudo do miocárdio (IAM) e acidente vascular encefálico (AVE) foram fatores de risco selecionados. Diabetes melito foi definido como autorreferido, acrescido ou não de terapia hipoglicemiante ou através da glicemia. Critério diagnóstico: glicemia de jejum $\geq 126 \mathrm{mg} / \mathrm{dL}$ e casu $\mathrm{al} \geq 200 \mathrm{mg} / \mathrm{dL}^{15}$. Na análise do diabetes melito e DAP sintomática, considerou-se o critério diagnóstico acima e terapia hipoglicemiante. Em relação à hipertensão arterial sistêmica, o critério foi: pressão sistólica $\geq 140 \mathrm{mmHg}$ e/ou diastólica $\geq 90 \mathrm{mmHg}^{16}$, ou se controlada através de anti-hipertensivos ou autorreferida.

Para o índice de massa corporal $\left(\mathrm{IMC}=\mathrm{kg} / \mathrm{m}^{2}\right)$, o critério foi: $\geq 19 ; 20-24,9 ; 25-29,9 \mathrm{e} \geq 30 \mathrm{~kg} / \mathrm{m}^{2}$, considerando como sobrepeso 25-29,9, e obesidade $\geq 30^{17}$, assim como para circunferência abdominal (traduzindo adiposidade visceral): $\geq 102 \mathrm{~cm}$ (homens) $\mathrm{e} \geq 88 \mathrm{~cm}$ (mulheres) ${ }^{18}$.

O ITB, sendo a razão entre a mais elevada pressão sistólica do tornozelo (dorsal do pé ou tibial posterior) e a mais elevada pressão sistólica dentre as braquiais, após repouso em decúbito dorsal por 5 minutos $^{1,2}$, foi aferido com um fluxômetro ultrassônico Parks Eletronics ${ }^{\circledR}$, com sonda de 8,5 MHz. Avaliou-se: braquiais, dorsais do pé e tibiais posteriores, separadamente, calculadas para cada extremidade, definindo como DAP $<0,90$, considerando-se o menor valor. A análise foi estratificada do seguinte modo: normal, 0,90-1,30 (> 1,30 artéria não-compressível); com DAP, subdividida em: estenose incipiente $(<0,90-0,80)$, discreta $(0,79-0,70)$, moderada $(0,69-0,50)$ e grave $(<0,50)$. Tal análise foi adaptada do critério diagnóstico da American Diabetes Association (ADA) ${ }^{3}$.

O questionário padronizado adaptado do San Diego Claudication Questionnaire ${ }^{19}$ identificou a prevalência de claudicação intermitente no membro inferior, categorizando claudicação clássica e/ou impotência funcional ao deambular na ausência de dor.

DAP sintomática: ITB $<0,90$ e claudicação; ITB $<0,90$ assintomática com ausência de claudicação, ambas com graduação de gravidade de acordo com a redu- ção do ITB, e seu grupo-controle, sem DAP e ITB $0,90-1,30^{4}$.

A análise estatística foi realizada com o programa Statistical Package for the Social Sciences (SPSS). Utilizou-se os testes de Mann-Whitney, quando a variável em questão esteve mensurada pelo menos em nível ordinal. Para a avaliação dos fatores de morbidade, utilizou-se o risco relativo (RR). Quando em nível nominal, recorreu-se ao qui-quadrado de Mantel-Haenszel, e, quando este se revelava inválido, reduziu-se a ordem das tabelas, ou recorreu-se ao teste exato de Fisher, no caso de tabelas redutíveis (2x2). Odds ratio (OR), ajustada com o intervalo de confiança de 95\% (IC95\%), foi estimada com modelo de regressão logística na análise dos fatores de risco e patologias associadas. A significância estatística foi considerada como $\mathrm{p}<0,05$.

\section{Resultados}

Foram selecionados 407 pacientes aferidos pelo ITB $<0,90$ com DAP $(n=248)$ e ITB $0,90-1,30$ sem DAP ( $\mathrm{n}=159)$, com predomínio do sexo feminino $(54 \%)$ e média de idade de 70,1 $\pm 10,2$ e $65,8 \pm 10,4$ anos, nos com e sem DAP, respectivamente $(\mathrm{p}<0,001)$. A estimativa da prevalência foi obtida pontualmente e também por IC95\% (com ITB < 0,90), sendo de 60,9 (56-66). Foram agrupadas as classes: sintomática, com $89,9 \%$ $(86,2-93,7)$, decorrente de isquemia crítica, com $32,2 \%$ (26,4-38,1), e assintomática, com $10,1 \%(6,3-13,8)$. A sintomática com claudicação típica representou, portanto, nove vezes mais que a assintomática. Já a isquemia crítica (ITB $<0,50$, subgrupo da sintomática) correspondeu a $32 \%$ desses pacientes. Ressalta-se a importância da detecção de assintomáticos com prevalência significativa, em população assistida numa unidade hospitalar universitária. Na Figura 2, analisou-se a prevalência ajustada por sexo e idade, estimativa que aumenta com faixa etária mais avançada, em ambos os sexos.

Entre os pacientes com 55-74 e os com 30-54 anos, a elevação nos primeiros foi de 20 e de 18 vezes no sexo masculino e feminino, respectivamente. Entretanto, essa relação se inverte nos pacientes $>74$ anos, com predomínio do sexo feminino na proporção de 1,35:1. As características demográficas e antropométricas associadas à sintomática, assintomática, e sem DAP foram ilustradas na Tabela 1. 


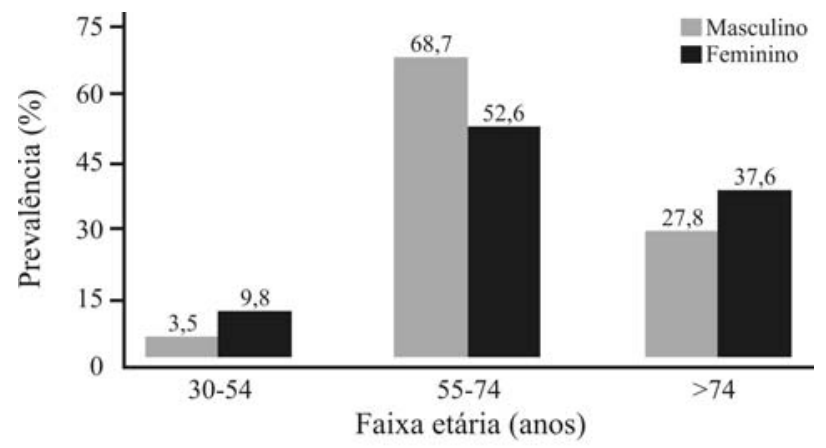

DAP: $\mathrm{n}=248 ;$ Sem DAP: $\mathrm{n}=159$.

Figura 2 - Prevalência da DAP em adultos $\geq 30$ anos, de acordo com ITB $<0,90$, ajustada por sexo e idade

A influência da faixa etária de 55-74 anos, 59,6 e $64 \%$ nos sintomáticos e assintomáticos, respectivamente, como também do sexo masculino, se manifesta como significativa ( $\mathrm{p}<0,001$ ), o que não ocorreu com o atributo cor. O sobrepeso, por sua vez, se associou positivamente à prevalência, o que não ocorreu com a adiposidade visceral. E o IMC aumenta a prevalência em mais de cinco vezes a partir de 19,9 a 25-29,9, enquanto que para o diabetes melito, a prevalência se eleva em uma vez e meia, quando comparada aos sem DAP.

As variáveis expostas na Tabela 2, analisando antecedentes mórbidos, hipertensão arterial sistêmica confirmada e diabetes melito, foram explicitadas tanto nos sintomáticos quanto nos assintomáticos, comparadas aos sem DAP, com seus respectivos RR, com todas influindo significativamente na prevalência da DAP $(p<0,001)$.

Destacou-se o RR do tabagismo influenciando o ITB $<0,90$, tanto na sintomática quanto na assintomática, sendo duas vezes maior do que nos não-fumantes. Tradicionais fatores de risco cardiovasculares estiveram associados à DAP, variando a OR de 2,03, 2,36, 2,74 e 3,30 para hipertensão autorreferida, IAM, hipertensão arterial sistê- mica confirmada e AVE, com seus respectivos IC95\%, como exibido na Figura 3.

Mostrou-se uma OR para DAP variando de duas a três vezes, excetuando-se o tabagismo, que contribuiu com um risco seis vezes maior. Em relação às médias e desvios padrão dos fatores de risco selecionados e ao ITB, a análise está contida na Tabela 3.

DAP sintomática e assintomática apresentaram idades mais avançadas, $71 \pm 10$ e $69 \pm 13$ anos, respectivamente, quando comparadas aos sem DAP, $65,8 \pm 10,4$ anos, com influência significativa $(p<0,001)$. Concernente às glicemias, tal diferença não é tão evidente, apesar de significativamente associada ao grupo sintomático, assintomático e sem DAP $(p<0,025)$. Pressões arteriais sistólicas e diastólicas se associaram a sintomática e assintomática, confrontadas com os sem DAP ( $p<0,001)$. Referente ao ITB, a média é mais baixa nos sintomáticos $(0,57 \pm 0,17)$ do que nos assintomáticos $(0,7 \pm 0,16)(\mathrm{p}<0,001)$. Percebe-se a

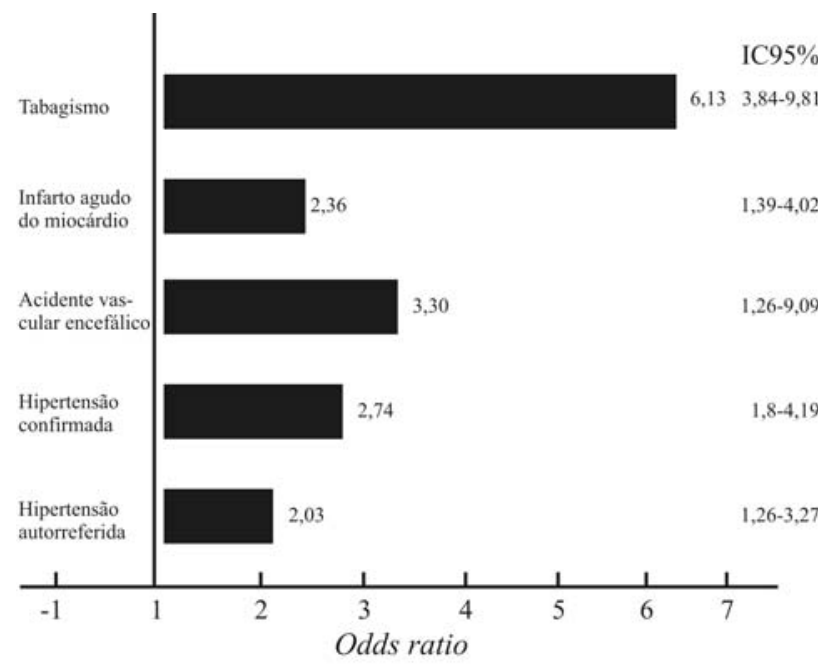

IC95\% = intervalo de confiança de $95 \%$.

Figura 3 - Odds ratio para DAP por fatores selecionados

Tabela 1 - Pacientes com e sem DAP segundo características demográficas e antropométricas

\begin{tabular}{lcccc}
\hline Características & \multicolumn{2}{c}{ DAP $(\mathrm{n}=248)$} & Sem DAP $(\mathrm{n}=159) \mathrm{n}(\%)$ & $\mathrm{p}\left(\chi^{2}\right)$ \\
\cline { 2 - 3 } & \multicolumn{1}{c}{ Sintomática $(\mathrm{n}=223) \mathrm{n}(\%)$} & Assintomática $(\mathrm{n}=25) \mathrm{n}(\%)$ & \\
\hline Sexo masculino & $107(48,0)$ & $8(32)$ & $55(34,6)$ & $<0,019$ \\
Idade de 55 a 74 anos & $133(59,6)$ & $16(64)$ & $112(70,4)$ & $<0,001$ \\
IMC de 25 a $29,9 \mathrm{~kg} / \mathrm{m}^{2}$ & $88(39,5)$ & $9(36)$ & $66(41,5)$ & $<0,004$ \\
\hline
\end{tabular}

$\mathrm{DAP}=$ doença arterial periférica; $\mathrm{IMC}=$ índice de massa corporal. 
Tabela 2 - Pacientes com e sem DAP segundo antecedentes mórbidos, DM e hipertensão arterial

\begin{tabular}{|c|c|c|c|c|c|}
\hline \multirow[t]{2}{*}{ Características } & \multicolumn{2}{|c|}{$\mathrm{DAP}(\mathrm{n}=248)$} & \multirow{2}{*}{$\begin{array}{c}\text { Sem DAP }(\mathrm{n}=159) \\
\mathrm{n}(\%)\end{array}$} & \multirow[t]{2}{*}{ RR (IC95\%) } & \multirow{2}{*}{$\mathrm{p}\left(\chi^{2}\right)$} \\
\hline & $\begin{array}{c}\text { Sintomática }(\mathrm{n}=223) \\
\mathrm{n}(\%)\end{array}$ & $\begin{array}{c}\text { Assintomática }(\mathrm{n}=25) \\
\mathrm{n}(\%)\end{array}$ & & & \\
\hline Hipertensão* & $177(80,1)$ & $18(72)$ & $103(65,6)$ & $1,34(1,02-1,70)$ & $<0,002$ \\
\hline AVE & $27(12,2)$ & $2(8,3)$ & $6(3,8)$ & $1,40(1,10-1,60)$ & $<0,005$ \\
\hline IAM & $73(33)$ & $6(25)$ & $26(16,4)$ & $1,35(1,14-1,55)$ & $<0,001$ \\
\hline Fumante/ex-fumante & $162(73)$ & $13(52)$ & $113(71,1)$ & $2,03(1,65-2,43)$ & $<0,001$ \\
\hline $\mathrm{DM}(\mathrm{mg} / \mathrm{dL})$ & $88(30,9)$ & $6(24,0)$ & $19(19,6)$ & $1,13(1,01-1,26)$ & $<0,025$ \\
\hline Hipertensão (mmHg) & $168(75,3)$ & $19(76)$ & $84(52,8)$ & $1,53(1,26-1,90)$ & $<0,001$ \\
\hline
\end{tabular}

$\mathrm{AVE}=$ acidente vascular encefálico; DAP $=$ doença arterial periférica; $\mathrm{DM}=$ diabetes melito na DAP sintomática, segundo glicemia de jejum $\geq 126 \mathrm{mg} / \mathrm{dL}$ ou casual $\geq 200 \mathrm{mg} / \mathrm{dL}$ e terapia hipoglicemiante; IAM = infarto agudo do miocárdio; IC95\% = intervalo de confiança de $95 \% ; \mathrm{RR}=$ risco relativo

* Autorreferida.

Tabela 3 - Médias e desvios padrão dos fatores de risco selecionados e do ITB

\begin{tabular}{|c|c|c|c|c|}
\hline \multirow[t]{2}{*}{ Características } & \multicolumn{2}{|c|}{$\mathrm{DAP}(\mathrm{n}=248)$} & \multirow{2}{*}{$\begin{array}{c}\text { Sem DAP }(n=159) \\
\text { Média }(\mathrm{DP})\end{array}$} & \multirow[t]{2}{*}{$\mathrm{p}\left(\chi^{2}\right)$} \\
\hline & $\begin{array}{c}\text { Sintomática }(\mathrm{n}=223) \\
\text { Média (DP) }\end{array}$ & $\begin{array}{c}\text { Assintomática }(\mathrm{n}=25) \\
\text { Média (DP) }\end{array}$ & & \\
\hline Idade (anos) & $71(10)$ & $69(13)$ & $65,8(10,4)$ & $<0,001$ \\
\hline Glicemia (mg/dL) & $124(53,3)$ & $116(38)$ & $117,5(47,3)$ & $<0,025$ \\
\hline PA sistólica (mmHg) & $156(28)$ & $150(41)$ & $138,6(23,2)$ & $<0,002$ \\
\hline PA diastólica (mmHg) & $88(10)$ & $85(13)$ & $83,9(7,3)$ & $<0,002$ \\
\hline ITB & $0,57(0,17)$ & $0,70(0,16)$ & $1,04(0,12)$ & $<0,000$ \\
\hline
\end{tabular}

$\mathrm{DAP}=$ doença arterial periférica; DP = desvio padrão; ITB = índice tornozelo-braquial; PA = pressão arterial.

concordância entre as duas categorias de DAP, analisadas de acordo com a adaptação do San Diego Claudication Questionnaire $^{19}$, apontando para a correlação entre as médias mais baixas do ITB e a presença ou ausência de claudicação, com significância em relação à média mais elevada do ITB nos sem DAP $(p<0,001)$.

As Figuras 4A e 4B expõem a análise por regressão logística da associação da pressão arterial sistólica e das glicemias, com a variável dependente ITB. Percebe-se relação inversamente proporcional, ou seja, quanto mais elevada a pressão arterial sistólica (150-180), menor o ITB $(0,60$ a 0,40$)(\mathrm{p}<0,001)$.

Quanto às glicemias, os valores estão mais baixos e mais agrupados, em torno de $125-130 \mathrm{mg} / \mathrm{dL}$, com ITB entre 0,70 a $0,40(\mathrm{p}<0,025)$.

A Figura 4C exibe a análise da estratificação do ITB por grau de severidade nos claudicantes ou não. As frequências apontam um quadro claro e consistente da DAP: leve intensidade em 13,7\%; discreta em 10,9\%; moderada em $43,5 \%$; e grave em $31,8 \%$. Considerando as categorias de DAP ordenadas pelo intervalo decrescente do ITB, registrou-se associação entre as mesmas, com correlação significativa para a moderada $(<0,70-0,50)(\mathrm{p}<0,001)$.

\section{Discussão}

Hirsch et al. ${ }^{20}$ mostraram que a DAP é facilmente detectável e diagnosticada pelo ITB $<0,90$ na assistência primária, com prevalência de $29 \%$ e subnotificação em mais de $50 \%$, propiciando complicações não tratadas adequadamente e no tempo ideal, elevando a morbidade, mortalidade cardiovascular e amputação de membro inferior. Estimaram que 8 a 12 milhões necessitarão do diagnóstico primário e intervenção clinica adequada.

Criqui et al. ${ }^{19}$ estudaram a correlação entre sintomas e o ITB para o diagnóstico da DAP utilizando o San Diego Claudication Questionnaire. Os sintomas resultaram da claudicação intermitente evoluindo para isquemia crítica. Os que obtiveram melhoria apresentaram um ITB muito próximo da normalidade, pelo desenvolvimento da circulação colate- 
A)

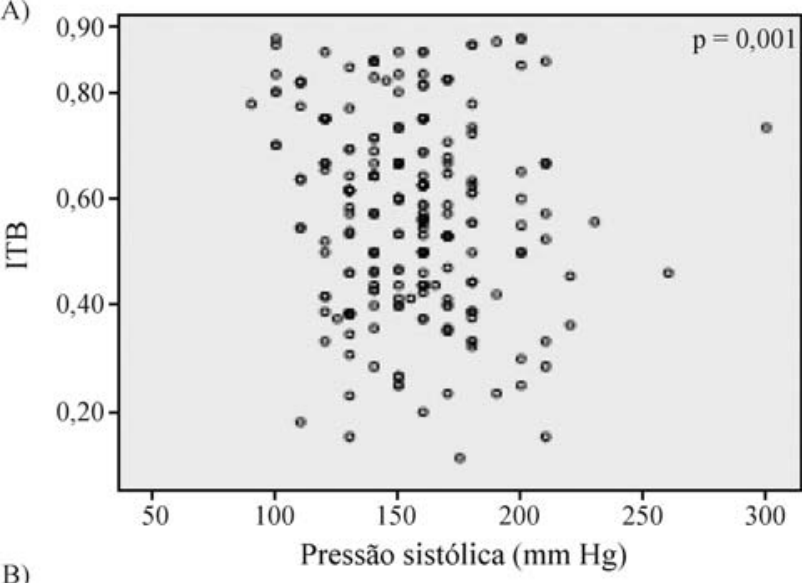

B)

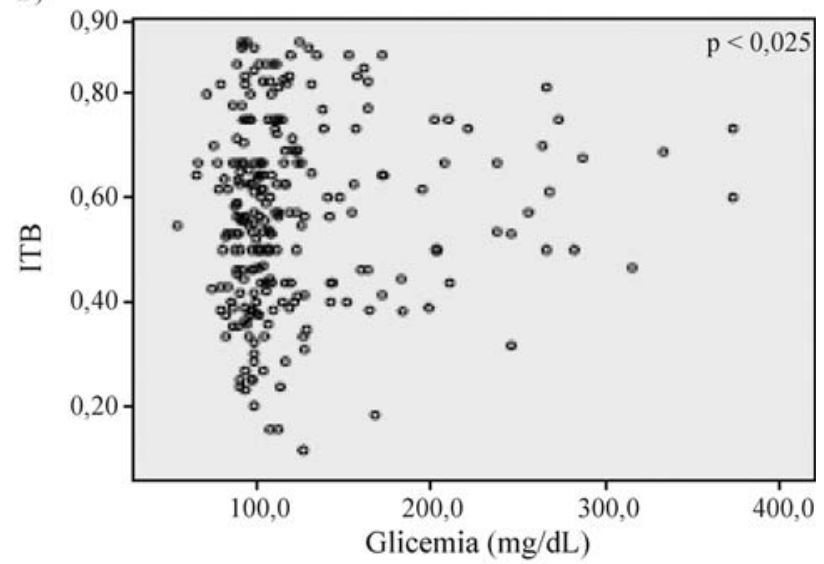

C)

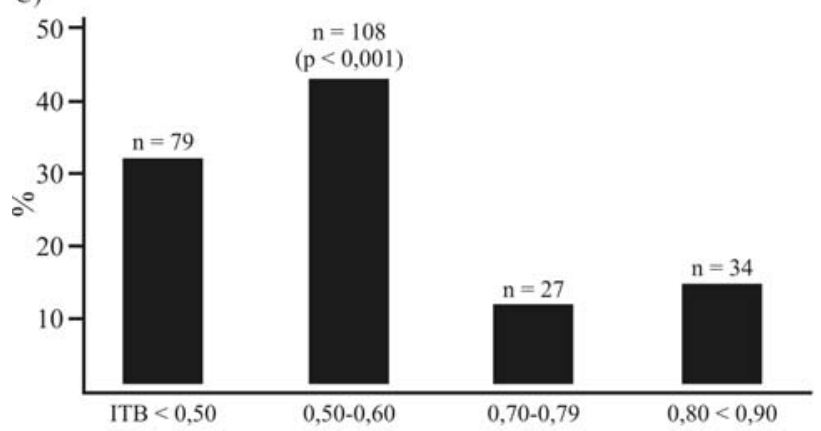

Distribuição de pacientes com DAP segundo intervalos do ITB $<0,90$

ITB $=$ indice tornozelo-braquial; DAP = doença arterial periférica.

Figura 4 - Regressão logística entre a variável dependente ITB $<0,90$

ral suficiente, não sendo encaminhados para avaliação clinica ou laboratorial, configurando a subnotificação.

Na população em geral, 10\% apresentaram sintomas clássicos de claudicação intermitente, $40 \%$ não os apresentaram e os outros $50 \%$ referiram sintomas atípicos ${ }^{4,13}$. McDermott et al. ${ }^{21}$ relataram que, numa população de mulheres idosas, a DAP assintomática está independentemen- te associada com a função dos membros inferiores. Lange et al. $^{22}$, por sua vez, discutiram diferentes metodologias, utilizando duplex scan na fossa antecubital, para determinação da pressão arterial sistólica, e nas artérias tibiais anteriores e posteriores, diversificando os critérios para o cálculo do ITB:

1) A maior pressão arterial tibial acima do tornozelo.

2) Somente a pressão sistólica da tibial posterior.

3) Somente a pressão sistólica da tibial anterior ou dorsal.

4) Após exercício, somente na artéria tibial posterior.

5) Expressaram a diferença de $10 \mathrm{mmHg}$ entre os membros superiores, sugerindo que o maior valor deva ser utilizado para a determinação do ITB.

Também estudaram a prevalência na Alemanha, em 34 centros primários, avaliando 50.000 indivíduos. Ressaltaram a DAP em 9,2\%, incluindo os claudicantes $(4,6 \%)$, comparando-os com o questionário Rose $(2,3 \%)^{22}$. Utilizando os cinco métodos descritos, mostraram prevalências variando entre $18,34,5,29,24,2$, e $27,8 \%$, respectivamente. Outra influência na prevalência é se o centro é primário, secundário ou terciário. Nunes et al. ${ }^{23}$, num centro terciário, apresentaram prevalência de $34,3 \%$ em pacientes encaminhados para angiografia coronariana. Os resultados do presente estudo mostraram uma prevalência de $60,9 \%$, ou seja, o dobro da referida pelos estudos de Nunes et al. ${ }^{23}$ e Lange et al. ${ }^{22}$, que somente aferiram a pressão sistólica da artéria tibial posterior. A prevalência elevada deste estudo pode ser decorrente, possivelmente, do fato de se tratar de um centro terciário universitário específico, e de os pacientes encaminhados para avaliação clínica serem tabagistas e já apresentarem doenças cardiovasculares prévias.

Esta amostra estimou assintomáticos em 10,1\%; sintomáticos, 89,9\%; e destes, com isquemia crítica, 32,2\%; enquanto que Bhatt et al. ${ }^{24}$ registraram que, apesar do risco e da prevalência, apenas $25 \%$ estão em tratamento.

McDermott et al. ${ }^{25}$ relataram que 8 milhões de americanos apresentam DAP com a clássica manifestação de claudicação intermitente. Entretanto, quando se utiliza o ITB $<0,90$, 20 a 50\% são assintomáticos, destacando que o significado clínico dos assintomáticos ainda não está esclarecido.

Na casuística do presente estudo, encontrou-se 10,1\% com ITB $<0,90$, sem claudicação, o que representa a meta- 
de dos $20 \%$ referidos por McDermott et al. ${ }^{25}$, possivelmente por se tratar de um centro terciário. Outra observação é que os assintomáticos neste trabalho apresentaram um ITB de $0,70 \pm 0,16$, similar aos $0,63 \pm 0,16$ referidos por McDermott et al. ${ }^{25}$ e descritos como próximo da normalidade por Criqui et al. ${ }^{19}$, enquanto que nos sintomáticos com claudicação intermitente encontramos um ITB menor $(0,57 \pm 0,17)$.

Nos assintomáticos, possivelmente ocorreu o desenvolvimento de circulação colateral suficiente, além da limitação de sua atividade laborativa, com diminuição da qualidade de vida. Os resultados desta pesquisa apontam que os assintomáticos não apresentam uma condição benigna, visto que referiram IAM e AVE pregressos em 12,2 e $8,3 \%$. No entanto, os fatores de risco cardiovasculares são similares. Diabetes melito e tabagismo influenciaram significativamente a DAP ${ }^{19}$. No presente estudo, o tabagismo influenciou o ITB $<0,90$ tanto na assintomática quanto na sintomática, com RR e OR, duas e seis vezes mais do que nos não-fumantes, respectivamente. A hipertensão arterial autorreferida, a confirmada, o IAM e o AVE apresentaram OR de 2,03, 2,74, 2,36 e 3,30, respectivamente.

Muitos estudos sugerem que a prevalência é similar entre homens e mulheres ${ }^{3-9}$. Nesta casuística, o sexo feminino prevaleceu apenas nos indivíduos $>74$ anos, numa relação de 1,35:1, provavelmente pela maior sobrevida nessa faixa etária.

A ADA $^{3}$ referiu prevalência de 20 e $29 \%$ em pacientes com DAP e diabetes, acima de 40 e 50 anos, respectivamente. Neste estudo, a prevalência da DAP com diabetes melito se eleva uma vez e meia, quando comparada à população sem DAP. No entanto, tal diferença não é tão evidente, apesar de significativamente associada à DAP sintomática e assintomática.

Dolan et al. ${ }^{26}$ estudaram 460 pacientes com DAP pelo ITB e questionário, sendo que 147 desses apresentavam diabetes e neuropatia, observando velocidade de $4 \mathrm{~m}$ durante 6 minutos de deambulação. A média do ITB foi similar nos com DAP com ou sem diabetes. No entanto, diabéticos eram mais jovens, apresentavam IMC mais elevado, neuropatia e mais eventos cardiovasculares, quando comparados aos com DAP apenas. Os diabéticos apresentaram menos sintomas clássicos de claudicação intermitente, deambularam a uma distância e velocidade menores, possivelmente pela neuropatia, quando comparados aos sem diabetes melito ${ }^{26}$.

$\mathrm{Na}$ presente pesquisa, os resultados com IMC $25-29,9 \mathrm{~kg} / \mathrm{m}^{2}$ sugerem que o sobrepeso se associou positivamente à prevalência de DAP, o que não ocorreu com a adiposidade visceral.

Selvin et al. ${ }^{27}$ avaliaram 2.174 participantes acima de 40 anos com ITB $<0,90$, para diagnóstico de DAP. Encontraram uma prevalência de 4,3\% (IC95\% 3,1-5,5), o que corresponde a cerca de 5 milhões de indivíduos (IC95\% 4 a 7 milhões). No entanto, nos indivíduos $>70$ anos foi de 14,5\% (IC95\% 10,8-18,2). Ressaltaram, ainda, que mais de $95 \%$ apresentaram pelo menos um dos fatores de risco cardiovascular. Estudos epidemiológicos mostraram que aproximadamente 2 a $3 \%$ dos homens e 1 a $2 \%$ das mulheres $\geq 60$ anos apresentam claudicação leve ou modera$\mathrm{da}^{4,5,14}$. A prevalência aumenta com a idade, e aproximadamente $20 \%$ dos acima de 70 anos apresenta DAP ${ }^{28}$. Esses pacientes irão desenvolver as complicações decorrentes das oclusões vasculares periféricas. Após 5 a 10 anos, um terço dos claudicantes evoluem para isquemia crítica, aproximadamente $20 \%$ requerem procedimento vascular, e 10\%, amputação primária de membro inferior ${ }^{4,5,10,11,29}$.

Mohler III ${ }^{14}$, em estudo prognóstico, refere que o ITB apresenta correlação com o grau de evolução dos sintomas da DAP, com eventos cardiovasculares e mortalidade cerebrovascular.

\section{Conclusão}

Este estudo aponta para a importância da introdução do ITB no diagnóstico da DAP, com configuração de graus de obstrução leve, discreta, moderada e grave para os sintomáticos e a identificação dos assintomáticos, possibilitando intervenção para os fatores de risco demarcados e o controle das complicações destes agravos.

\section{Referências}

1. McDermott MM, Criqui MH, Liu K, et al. Lower ankle/brachial index, as calculated by averaging the dorsalis pedis and posterior tibial arterial pressures, and association with leg functioning in peripheral arterial disease. J Vasc Surg. 2000;32:1164-71.

2. Fowkes F, Lee A, Murray G. On behalf of the ABI collaboration. Ankle-Brachial Index as an independent indicator of mortality of fifteen international population cohort studies. Circulation. 2005;112:3704. 
3. American Diabetes Association (ADA). Peripheral arterial disease in people with diabetes. Diabetes Care. 2003;26:3333-41.

4. Hirsch AT, Haskal ZJ, Hertzer NR, et al. ACC/AHA 2005 practice guidelines for the management of patients with peripheral arterial disease (lower extremity, renal, mesenteric, and abdominal aortic): a collaborative report from the American Association for Vascular Surgery/Society for Vascular Surgery, Society for Cardiovascular Angiography and Interventions, Society for Vascular Medicine and Biology, Society of Interventional Radiology, and the ACC/AHA Task Force on Practice Guidelines (writing Committee to Develop Guidelines for the Management of Patients With Peripheral Arterial Disease): endorsed by the American Association of Cardiovascular and Pulmonary Rehabilitation; National Heart, Lung, and Blood Institute; Society for Vascular Nursing; TransAtlantic Inter-Society Consensus; and Vascular Disease Foundation. Circulation. 2006;113:463-654.

5. Weitz JI, Byrne J, Clagget GP, et al. Diagnosis and treatment of chronic arterial insufficiency of the lower extremities: a critical review. Circulation. 1996;94:3026-49.

6. TransAtlantic Inter-Society Consensus Revascularization. Management of the peripheral arterial disease (PAD). Eur J Endovasc Surg. 2000,19 (Suppl A):S1-S250.

7. TransAtlantic Inter-Society Consensus (TASC). Management of the peripheral arterial disease (PAD). Int Angiol. 2000;19 (1 Suppl 1):1-XXIV;1-304.

8. Dormandy JA, Rutherford RB. Management of peripheral arterial disease (PAD). TASC Working Group. TransAtlantic Inter-Society Consensus (TASC). J Vasc Surg. 2000;31:S1-S296.

9. Norgren L, Hiatt WR, Dormandy JA, et al. Inter-Society Consensus for the management of Peripheral Arterial Disease (TASC II) J Vasc Surg. 2007;45 (Suppl 1):S5-S67.

10. Spichler ER, Spichler D, Lessa I, Costa e Forti A, Franco LJ, LaPorte RE. Capture-recapture method to estimate lower extremity amputation rates in Rio de Janeiro, Brazil. Rev Panam Salud Publica. 2001;10:334-40.

11. Spichler D, Miranda Jr. F, Spichler ES, Franco LJ. Major lower extremity amputations related to peripheral arterial disease and diabetes mellitus in the city of Rio de Janeiro. J Vasc Bras. 2004;3:111-22.

12. Lijmer JG, Hunink MG, van den Dungen JJ, Loonstra J, Smit AJ. ROC analysis of noninvasive tests for peripheral arterial disease. Ultrasound Med Biol. 1996;22:391-8.

13. Criqui MH, Denenberg JO, Langer RD, Fronek A. The epidemiology of peripheral arterial disease: importance of identifying the population at risk. Vasc Med. 1997;2:221-6.

14. Mohler III ER. Peripheral Arterial Disease. Curr Treat Options Cardiovasc Med. 1999;1:27-34.

15. American Diabetes Association. Diagnosis and classification of diabetes mellitus. Diabetes Care. 2008;31:S55-60.

16. Williams B, Poulter NR, Brown MJ, et al. Guidelines for management of hypertension: report of the fourth working party of the British Hypertension Society, 2004-BHS IV. J Hum Hypertens. 2004;18:139-85.

17. Executive summary of the clinical guidelines on the identification, evaluation, and treatment of overweight and obesity in adults. Arch Intern Med. 1998;158:1855-67.
18. Alberti KG, Zimmet P, Shaw J. Metabolic syndrome- a new world-wide definition. A Consensus Statement from the International Diabetes Federation. Diabet Med. 2006;23:469-80.

19. Criqui MH, Denenberg JO, Bird CE, Fronek A, Klauber MR, Langer RD. The correlation between symptoms and noninvasive test results in patients referred for peripheral arterial disease testing. Vasc Med. 1996;1:65-71.

20. Hirsch AT, Criqui MH, Treat-Jacobson D, et al. Peripheral arterial disease detection, awareness, and treatment in primary care. JAMA. 2001;286:1317-24.

21. McDermott MM, Fried L, Simonsick E, Ling S, Guralnik JM. Asymptomatic peripheral arterial disease is independently associated with impaired lower extremity functioning: the women's health and aging study. Circulation. 2000;101:1007-12. Erratum in: Circulation. 2001;104:504.

22. Lange SF, Trampisch HJ, Pittrow D, et al. Profound influence of different methods for determination of the ankle brachial index on the prevalence estimate of peripheral arterial disease. BMC Public Health. 2007;7:147.

23. Nunes JL, Silvany-Neto A, Pitta GB, et al. Prevalence of peripheral arterial occlusive disease in patients referred to a tertiary care hospital in Salvador, Bahia, Brazil, for coronary angiography. Braz J Med Biol Res. 2008;41:202-8.

24. Bhatt DL, Steg PG, Ohman EM, et al. International prevalence, recognition, and treatment of cardiovascular risk factors in outpatients with atherothrombosis. JAMA. 2006;295:180-9.

25. McDermott MM, Guralnik JM, Ferrucci L, et al. Asymptomatic peripheral arterial disease is associated with more adverse lower extremity characteristics than intermittent claudication. Circulation. 2008;117:2484-91.

26. Dolan NC, Liu K, Criqui MH, et al. Peripheral artery disease, diabetes, and reduced lower extremity functioning. Diabetes Care. 2002;25:113-120.

27. Selvin E, Erlinger TP. Prevalence of and risk factors for peripheral arterial disease in the United States: results from the National Health and Nutrition Examination Survey, 1999-2000. Circulation. 2004;110:738-43.

28. Regensteiner JG, Hiatt WR, Coll JR, et al. The impact of peripheral arterial disease on health-related quality of life in the Peripheral Arterial Disease Awareness, Risk, and Treatment: New Resources for Survival (PARTNERS) Program. Vasc Med. 2008;13:15-24.

29. Dormandy J, Heeck L, Vig S. Predicting which patients will develop chronic critical leg ischemia. Semin Vasc Surg. 1999;12:138-41.

Correspondência:

Marilia Duarte Brandão Panico

Hospital Universitário Pedro Ernesto

Faculdade de Ciências Médicas - UERJ

Boulevard Vinte e Oito de Setembro, 77/3 $3^{\circ}$ andar,

Vila Isabel

CEP 20551-030 - Rio de Janeiro, RJ

Tel.: (21) 2208.6030, (21) 2587.6624, (21) 9621.4207

E-mail: mariliapanico@hotmail.com,

mariliapanico@uol.com.br 